TITLE:

\title{
Quark number holonomy and confinement-deconfinement transition
}

\section{AUTHOR(S):}

Kashiwa, Kouji; Ohnishi, Akira

\section{CITATION:}

Kashiwa, Kouji ... [et al]. Quark number holonomy and confinementdeconfinement transition. Physical Review D 2016, 93(11): 116002.

ISSUE DATE:

2016-06-01

URL:

http://hdl.handle.net/2433/233192

RIGHT:

(C) 2016 American Physical Society. 
PHYSICAL REVIEW D 93, 116002 (2016)

\title{
Quark number holonomy and confinement-deconfinement transition
}

\author{
Kouji Kashiwa* and Akira Ohnishi ${ }^{\dagger}$ \\ Yukawa Institute for Theoretical Physics, Kyoto University, Kyoto 606-8502, Japan
}

(Received 1 March 2016; published 6 June 2016)

\begin{abstract}
We propose a new quantity which describes the confinement-deconfinement transition based on topological properties of QCD. The quantity which we call the quark number holonomy is defined as the integral of the quark number susceptibility along the closed loop of $\theta$ where $\theta$ is the dimensionless imaginary chemical potential. The expected behavior of the quark number holonomy at finite temperature is discussed, and its asymptotic behaviors are shown.
\end{abstract}

DOI: 10.1103/PhysRevD.93.116002

\section{INTRODUCTION}

Understanding the confinement-deconfinement transition in quantum chromodynamics (QCD) is one of the interesting and important subjects in nuclear and elementary particle physics. In the heavy quark mass limit, spontaneous breaking of the center $\left(\mathbb{Z}_{N_{\mathrm{c}}}\right)$ symmetry is directly related to the confinement-deconfinement transition, where $N_{\mathrm{c}}$ is the number of color. Then, the holonomy which is the gaugeinvariant integral along the closed Euclidean temporal coordinate loop becomes an exact order parameter of the confinement-deconfinement transition. It is called the Polyakov loop. On the other hand, we cannot find any exact order parameters in the case with dynamical quarks at present, where the direct relation between $\mathbb{Z}_{N_{\mathrm{c}}}$ symmetry and the confinement-deconfinement transition is lost.

\section{A. Topological order}

The notion of topological order may be of great help in understanding the nature of the confinement-deconfinement transition. Recently, important progress was made in that the confined and deconfined states at zero temperature $(T=0)$ were mathematically classified based on the topological order [1] in Ref. [2]. Motivated by this progress, it has been suggested that the confinement-deconfinement transition can be described by using the analogy of the topological order and then that the free-energy degeneracy plays a crucial role [3]. The idea of topological order is extended to finite temperature in terms of the Uhlmann phase $[4,5]$. The Uhlmann phase is an extension of the Berry phase to mixed quantum states. The Uhlmann phase can describe the topological order at finite $T$ in the one-dimensional fermion systems such as the topological insulator and the superconductor [5]. The Uhlmann phase is defined by using the amplitude for the density matrix where amplitudes form the Hilbert space. There is the $U(n)$ gauge freedom of the amplitude where $n$ is the dimension of the space and it is a generalization of the $U(1)$ gauge freedom of pure quantum

\footnotetext{
*kouji.kashiwa@yukawa.kyoto-u.ac.jp

†ohnishi@yukawa.kyoto-u.ac.jp
}

states. At finite $T$, the Uhlmann phase includes information of the density matrix of the statistical mechanics and is calculated by the contour integral along the crystalline momentum. Unfortunately, the calculation of the Uhlmann phase in QCD seems to be very difficult or impossible at present.

\section{B. Imaginary chemical potential}

In QCD at finite $T$, imaginary chemical potential $\left(\mu_{\mathrm{I}}\right)$ is an external parameter, which shows periodicity; chemical potential $\mu$ appears in the form of fugacity $e^{\mu / T}=$ $e^{\mu_{\mathrm{R}} / T} e^{i \mu_{\mathrm{I}} / T}$ in the free energy, and the two states at $\mu_{\mathrm{I}}=0$ and $\mu_{\mathrm{I}}=2 \pi T$ are physically the same. In addition to this $2 \pi T$ periodicity, characteristic periodicity appears at finite imaginary chemical potential $\left(\mu_{\mathrm{I}}\right)$ and is called the Roberge-Weiss (RW) periodicity [6]. The RW periodicity has deep relations with the free-energy degeneracy and thus it is natural to expect that some hints to understand the confinement-deconfinement transition are hidden in the imaginary chemical potential region.

\section{Quark number holonomy}

We investigate the confinement-deconfinement transition by using the imaginary chemical potential in this paper. We discuss the contour integral along the closed loop of the imaginary chemical potential, $\mu_{\mathrm{I}}=0 \sim 2 \pi T$, or the dimensionless quark imaginary chemical potential, $\theta=\mu_{\mathrm{I}} / T=0 \sim 2 \pi$. Particularly, we focus on the behavior of the quark number density at finite $\mu_{\mathrm{I}}$ and propose a new quantity which can describe the confinementdeconfinement transition based on it. It is a new quantum order parameter of the confinement-deconfinement transition when dynamical quarks are acting in the system. We call it the "quark number holonomy." The quark number holonomy seems to be a similar quantity to the Uhlmann phase $[4,5]$. The quark number holonomy defined in Eq. (3) also includes the information of the density matrix via the quark number density and is calculated by the contour integral along the closed loop of $\theta$. It should be noted that the quark number holonomy can be calculated in the effective 
models of QCD and lattice QCD simulation as discussed later. It is the most important reason why we propose the new quantity for the confinement-deconfinement transition in this paper.

This paper is organized as following. In the next section, we propose new quantity which is so called the quark number holonomy. Some discussions for the quark number holonomy are shown in Sec. III. Section IV is devoted to summary.

\section{QUARK NUMBER HOLONOMY}

In this section, we firstly summarize QCD periodicities and special transitions which appear at finite $\mu_{\mathrm{I}}$. Secondly, we propose a new quantity which describes the confinement-deconfinement transition based on QCD properties at finite $\mu_{\mathrm{I}}$. Finally, the infinite $T$ and the infinite bare quark mass $(m)$ limit are discussed.

\section{A. QCD periodicities and transitions at finite $\mu_{I}$}

It is known that the QCD partition function $\left(Z_{\mathrm{QCD}}\right)$ has the RW periodicity [6],

$$
Z_{\mathrm{QCD}}(\theta)=Z_{\mathrm{QCD}}\left(\theta+\frac{2 \pi k}{N_{\mathrm{c}}}\right)
$$

where $k$ is any integer. It should be noted that the RW periodicity is a model independent and exact property of the QCD partition function. In the pure gauge limit, there is the $\mathbb{Z}_{N_{\mathrm{c}}}$ symmetry, but it is explicitly broken by dynamical quark contributions. The RW periodicity is nothing but the remnant of the $\mathbb{Z}_{N_{\mathrm{c}}}$ symmetry in the pure gauge limit. If we neglect the $\theta$ dependence of the gauge field through quark contributions, the RW periodicity is lost and then the partition function only has the trivial $2 \pi$ periodicity. This situation also appears in the quenched approximation.

In addition to the RW periodicity, QCD has special transition at $\theta=(2 k-1) \pi / N_{\mathrm{c}}$ which is called the RW transition. The RW periodicity is realized in a different way at the RW transition in the confined and deconfined phases. To discuss the RW transition, the phase of the Polyakov loop is useful. The Polyakov loop can be expressed as

$$
\Phi=\frac{1}{N_{\mathrm{c}}} \operatorname{tr} \mathcal{P}\left[\exp \left(i g \oint_{0}^{\beta} A_{4}(\tau, \vec{x}) d \tau\right)\right]=|\Phi| e^{i \phi},
$$

where $\beta$ is the inverse temperature $(\beta=1 / T), \mathcal{P}$ is the pathordering operator and $\phi$ is the Polyakov-loop phase. When $\theta$ is continuously changed from 0 to $2 \pi$, the phase of the Polyakov loop is smoothly rotated below $T_{\mathrm{RW}}$, but it becomes discontinuous above $T_{\mathrm{RW}}$ at $\theta=(2 k-1) \pi / N_{\mathrm{c}}$. Such $\theta$ dependence of $\phi$ can be found in Ref. [7] for the Polyakov-loop extended Nambu-Jona-Lasinio (PNJL) model [8] and Ref. [9-11] for lattice QCD. The endpoint of the RW transition is called the RW endpoint and its temperature is denoted by $T_{\mathrm{RW}}$.

\section{B. Deconfinement transition from RW periodicity}

In Ref. [3], the authors proposed the new classification of the confined and deconfined phases at finite $T$ based on the RW periodicity. The different realization of the RW periodicity plays a crucial role in the classification:

Confined phase. The origin of the RW periodicity is the dimensionless baryon chemical potential $3 \theta$ in the form of $\exp ( \pm 3 i \theta)$. For example, it can be seen from the strong coupling limit of QCD with the mean-field approximation; see Ref. [12,13].

Deconfined phase. The origin is the dimensionless quark chemical potential and the gauge field in the form of $\exp \left[ \pm i\left(g A_{4} / T+\theta\right)\right]$ where $\mathbb{Z}_{3}$ images are important [6]. It can be clearly seen in the perturbative one-loop effective potential $[14,15]$.

Therefore, in our approach for the investigation of the confinement-deconfinement transition, we focus on the response of the system against $\theta$ as an indicator of the nontrivial free-energy degeneracy. The system does not show singularities along $\theta$ at $\left(T, \mu_{\mathrm{R}}\right)$ in the confined phase. By comparison, there should be some singularities along $\theta$ at $\left(T, \mu_{\mathrm{R}}\right)$ in the deconfined phase. Details of singularities are explained in Sec. IIC; for example, see Fig. 1. Confinement-deconfinement transition temperatures determined by the nontrivial free-energy degeneracy and the Polyakov loop are matched with each other in the infinite quark mass limit. In the next subsection, we propose a new quantum order-parameter of the confinement-deconfinement transition based on the RW periodicity. In the following discussions in this section, we concentrate on the case with $\mu_{\mathrm{R}}=0$.

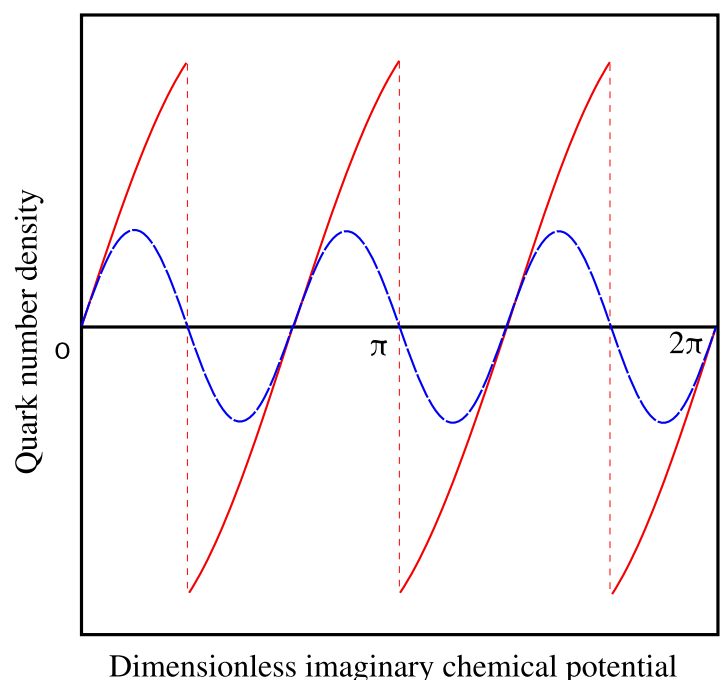

FIG. 1. The schematic behavior of $n_{q}$ as a function of $\theta$ for $N_{\mathrm{c}}=3$. The periodic solid and dashed lines represent the quark number density at $T \gg T_{\mathrm{RW}}$ and $T \ll T_{\mathrm{RW}}$, respectively. 


\section{Definition of quark number holonomy}

The quark number density $\left(n_{q}\right)$ above $T_{\mathrm{RW}}$ should have the gap at $\theta=(2 k-1) \pi / N_{\mathrm{c}}$ which reflects the $\theta$-odd property. The schematic behavior of $n_{q}$ with $N_{\mathrm{c}}=3$ is shown in Fig. 1. The periodic solid and dashed lines represents $n_{q}$ at sufficiently high and low $T$ comparing with $T_{\mathrm{RW}}$, respectively. By using the behavior of $n_{q}$ at finite $T$, we can construct the order parameter,

$$
\Psi(T)=\left[\oint_{0}^{2 \pi}\left\{\operatorname{Im}\left(\left.\frac{d \tilde{n}_{q}}{d \theta}\right|_{T}\right)\right\} d \theta\right]
$$

where $\tilde{n}_{q}$ is the normalized quark number density defined as $\tilde{n}_{q} \equiv C n_{q}$ here the coefficient $C\left[\mathrm{MeV}^{-3}\right]$ is introduced to make $\tilde{n}_{q}$ dimensionless. It becomes nonzero at $T \gg T_{\mathrm{RW}}$ and zero at $T \ll T_{\mathrm{RW}}$ because the information of the gap at $\theta=(2 k-1) \pi / N_{\mathrm{c}}$ is missed when we perform the differential calculus and the numerical integration. We call Eq. (3) the quark number holonomy. The integrand of Eq. (3) can be expressed as

$$
\operatorname{Im}\left(\left.\frac{d \tilde{n}_{q}}{d \theta}\right|_{T}\right)=-\left.\frac{C T^{2}}{V}\left[\langle\tilde{N}\rangle^{2}-\left\langle\tilde{N}^{2}\right\rangle\right]\right|_{T} \propto \chi_{2}^{q},
$$

where $V$ denotes the three-dimensional volume and the operator $\tilde{N}$ is $\int\left(q^{\dagger} q\right) d^{4} x$. In Eq. (4), $\chi_{2}^{q}$ is nothing but the quark number susceptibility at finite $\theta$. The expected behavior of the quark number holonomy as a function of $T$ is shown in Fig. 2. We assume that the RW endpoint is second (first) order in case A (B). The schematic phase diagram in case B is shown in the inset of Fig. 2. When $T_{\mathrm{RW}}$ is first order, the RW endpoint can have two more firstorder lines. In this paper, we call it the beard line, and the endpoint temperature of the beard line is denoted by $T_{\text {Beard. }}$

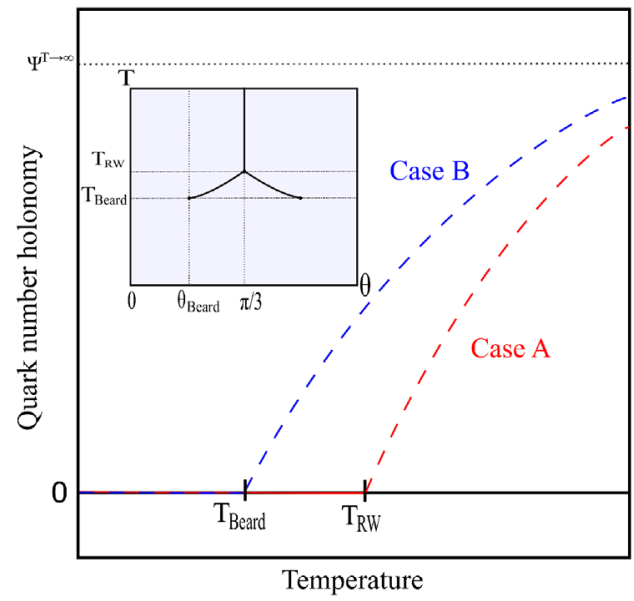

FIG. 2. The expected behavior of $\Psi$ as a function of $T$. In case A, the RW endpoint is second order, while it is the triple point in case B. The actual value of $\Psi^{T \rightarrow \infty}$ is explained in the text of Sec. II D. The inset figure shows the schematic phase diagram in case B with $N_{\mathrm{c}}=3$ as a function of $\theta$ and $T$.
This triple point scenario has been predicted by the lattice QCD simulations [16,17]. This behavior may be induced by the correlation between the chiral and deconfinement dynamics, but details are still under debate.

In case $\mathrm{A}$, where the RW endpoint is second order, the quark number holonomy can be expressed as

$$
\Psi= \pm 2 N_{\mathrm{c}} \lim _{\epsilon \rightarrow 0}\left[\operatorname{Im} \tilde{n}_{q}\left(\theta=\theta_{\mathrm{RW}}^{\mp}\right)\right],
$$

where $\theta_{\mathrm{RW}}^{\mp}=\theta_{\mathrm{RW}} \mp \epsilon=\pi / N_{\mathrm{c}} \mp \epsilon$ with the positive infinitesimal value $\epsilon$. Below $T_{\mathrm{RW}}, n_{q}\left(\theta=\pi / N_{\mathrm{c}}\right)$ is exactly zero and thus $\Psi=0$, but $\Psi$ becomes nonzero above $T_{\mathrm{RW}}$. The coefficient $N_{\mathrm{c}}$ in Eq. (5) reflects the number of the gapped point in the $0 \leq \theta \leq 2 \pi$ region.

In case $\mathrm{B}$ where the RW endpoint is the triple point, situations become complicated in the $T_{\text {Beard }}<T<T_{\mathrm{RW}}$ region. In this region, $\tilde{n}_{q}$ jumps from high- $T$ curve to the low- $T$ curve at $\theta=\theta_{\mathrm{B}}$ where $\theta_{\mathrm{B}}$ is defined as the dimensionless imaginary chemical potential on the beard line. The quark number holonomy can be expressed as

$$
\Psi=2 N_{\mathrm{c}} \lim _{\epsilon \rightarrow 0}\left[\operatorname{Im}\left\{\tilde{n}_{q}\left(\theta=\theta_{\mathrm{B}}^{-}\right)-\tilde{n}_{q}\left(\theta=\theta_{\mathrm{B}}^{+}\right)\right\}\right] \neq 0,
$$

where $\theta_{\mathrm{B}}^{\mp}$ mean $\theta_{\mathrm{B}} \mp \epsilon$. The number of the gapped point is $2 N_{\mathrm{c}}$ in the $0 \leq \theta \leq 2 \pi$ region above $T_{\text {Beard }}$. Below $T_{\text {Beard }}, \Psi$ should be zero.

\section{Asymptotic behavior}

Here, we discuss the asymptotic behavior of $\Psi$. The quark number holonomy in the $T \rightarrow \infty$ limit becomes

$$
\Psi^{T \rightarrow \infty}=2 N_{\mathrm{c}} \lim _{\epsilon \rightarrow 0}\left[\operatorname{Im} \tilde{n}_{q}^{T \rightarrow \infty}\left(\theta=\theta_{\mathrm{RW}}^{-}\right)\right] \neq 0,
$$

where $\tilde{n}_{q}^{T \rightarrow \infty}$ is the normalized quark number density in the $T \rightarrow \infty$ limit. Actual value of $\tilde{n}_{q}^{T \rightarrow \infty}$ can be obtained from the perturbative one-loop effective potential $[14,15,18]$ and the value becomes

$$
\begin{gathered}
\lim _{\epsilon \rightarrow 0} \frac{\operatorname{Im} \tilde{n}_{q}^{T \rightarrow \infty}\left(\theta=\theta_{\mathrm{RW}}^{\mp}\right)}{N_{\mathrm{c}} N_{\mathrm{f}}}= \pm \frac{2 \pi}{3}\left[\frac{\theta_{\mathrm{RW}}}{2 \pi}-4\left(\frac{\theta_{\mathrm{RW}}}{2 \pi}\right)^{3}\right] \\
\underset{N_{\mathrm{c}}=3}{\longrightarrow} \pm 0.31 \ldots,
\end{gathered}
$$

where the normalization constant $C$ is set to $T^{-3}$.

The quark number holonomy in the $m \rightarrow \infty$ limit can be discussed by using the hopping parameter expansion in the lattice formalism. From the straightforward calculation, the normalized quark number density is obtained as

$$
\begin{aligned}
\lim _{\epsilon \rightarrow 0}\left[\operatorname{Im} \tilde{n}_{q}\left(\theta=\theta_{\mathrm{RW}}^{-}\right)\right] \\
=-\frac{C N_{\mathrm{f}}}{N_{s}^{3} N_{t}} \lim _{\epsilon \rightarrow 0} \sum_{n=1}^{\infty} \kappa^{n} \operatorname{Im}\left\langle\operatorname{Tr}\left(\frac{\partial Q}{\partial \mu} Q^{n-1}\right)\right\rangle,
\end{aligned}
$$


where the hopping parameter $\kappa$ is related to $m$ as $\kappa=1 /(2 m+8), \quad N_{s}^{3} N_{t}$ express the space-time lattice volume, $\langle\cdots\rangle$ means the configuration average and $Q$ is the covariant derivative part of the lattice action; see Ref. [19] as an example. Since the hopping parameter is $\kappa \propto 1 / m$ in the heavy quark mass limit, the quark number holonomy is suppressed by $1 / m$. Thus, the quark number holonomy finally becomes zero at $m=\infty$. However, the confinement-deconfinement transition temperature determined by the quark number holonomy perfectly matches with the transition temperature determined by $\Phi$ at $m=\infty$ by carefully considering $m \rightarrow \infty$ limit.

Even in the heavy quark mass region, the RW transition may be smeared by the finite size effect in the lattice QCD simulation. Thus, the nonzero quark number holonomy requires finite size scaling analysis to obtain in a straightforward calculation. An alternative way is to fit lattice QCD data by using an oscillating $\theta$-odd functions in the region $V$, $\left\{V: 0 \leq \theta \leq \pi / N_{\mathrm{c}}\right\}$, with neglecting data very close to $\theta=$ $(2 k-1) \pi / N_{\mathrm{c}}$ where data are strongly affected by the finite size effects. The fitting function becomes the $2 \pi / N_{\mathrm{c}}$ periodic function at sufficiently low $T$ and it does the $2 \pi$ periodic function at sufficiently high $T$ in the $0 \leq \theta \leq \pi / N_{\mathrm{c}}$ region. This difference may help us to calculate the quark number holonomy on the lattice. It should be noted that this treatment is similar to the observation process of nonzero order-parameters with vanishing symmetry breaking external fields on the lattice and thus it is not a fundamental problem.

\section{DISCUSSIONS}

Firstly, we discuss the current status of the present determination and the ordinary determination of the deconfinement temperature. Readers may doubt the validity of the present definition of the deconfinement transition temperature since the deconfinement temperature, $T_{\mathrm{D}} \equiv T_{\mathrm{RW}}$ or $T_{\text {Beard }}$, is substantially higher than the chiral pseudocritical temperature. It was considered that the chiral and the deconfinement crossover take place at similar temperatures from the rapid change of the chiral condensate and the Polyakov loop on the lattice with $2+1$ flavors; see for example Ref. [20,21]. With the development of the highly improved quark action, it now seems that the Polyakov loop grows very gradually [22,23]. An effective model analysis of recent lattice data implies that the deconfinement pseudocritical temperature $(\sim 215 \mathrm{MeV})$ is substantially higher than the chiral pseudocritical temperature [24]. By comparison, a recent lattice determination of the RW endpoint temperature with physical quark masses implies that the continuum extrapolated value of $T_{\mathrm{RW}}$ is 208(5) MeV [25]. Therefore, higher $T_{\mathrm{D}}$ does not invalidate the discussion, but is supported by the recent lattice data via effective model analysis.

Secondly, we discuss the difference between the quark number holonomy and the dual quark condensate [26-30]. The dual quark condensate is defined as
$\Sigma^{(n)}=-\oint_{0}^{2 \pi} \frac{d \varphi}{2 \pi} e^{-i n \varphi} \sigma(\varphi)$

where $\varphi=\theta+\pi$ specifies the boundary condition for the temporal direction of quarks, $\sigma(\varphi)$ is the $\varphi$-dependent chiral condensate and $n$ represents the winding number along the temporal direction. In particular, $\Sigma^{(1)}$ shares similar properties with $\Phi$ because $\Phi$ is also the winding number 1 quantity and, thus, it can be used as the indicator of the confinement-deconfinement transition. In the quenched approximation, the dual quark condensate is well defined, but there is the uncertainty in the dynamical quark case $[27,28]$. In the calculation of the dual quark condensate, we need to break the RW periodicity because $\Sigma^{(1)}$ should be zero in all $T$ region if the RW periodicity exists. It is usually done by imposing the twisted boundary condition on the Dirac operator, while configurations are sampled under the antiperiodic boundary condition. This is not a unique procedure. Therefore, there is the uncertainty in the determination of the dual quark condensate. Also, it is well known that the dual quark condensate is strongly affected by the chiral transition or some other transitions [31-33]. On the other hand, the quark number holonomy (3) can provide nonzero value above $T_{\mathrm{RW}}$ or $T_{\text {Beard }}$ without any uncertainties. It is the important advantage of the quark number holonomy.

Third, we discuss the quark number holonomy from the landscape of the effective potential in the complex $\Phi$ plane. Below $T_{\mathrm{RW}}$ or $T_{\text {Beard }}$, the effective potential at any $\theta$ can be described by only one minimum which is continuously connected with the $\theta=0$ solution. Above $T_{\mathrm{RW}}$, the $\mathbb{Z}_{N_{\mathrm{c}}}$ images appear; for example, the $\mathbb{Z}_{N_{\mathrm{c}}}$ images are $e^{i 2 \pi / 3}$ and $e^{i 4 \pi / 3}$ for $\Phi=1$ at sufficiently high $T$ for $N_{\mathrm{c}}=3$. In the confined phase, the fluctuation is strong and thus the $\mathbb{Z}_{N}$. images are collapsed to one minimum. On the other hand, the $\mathbb{Z}_{N_{\mathrm{c}}}$ images can withstand the fluctuation in the deconfined phase. Thus, the quark number holonomy (3) measures the strength of the fluctuation which collapses the $\mathbb{Z}_{N_{\mathrm{c}}}$ images to one minimum. It is related to the nontrivial degeneracy of the free energy in the deconfined phase discussed in Ref. [3]. Therefore, the quark number holonomy can describe the confinement-deconfinement transition via the nontrivial free-energy degeneracy. The present discussion may be related to the Polyakov-loop fluctuations discussed in Ref. [34] and, thus, it is interesting to compare the results of the Polyakov-loop fluctuations with the quark number holonomy.

Finally, the sign problem is discussed when we calculate the quark number holonomy at finite $\mu_{\mathrm{R}}$. At finite $\mu_{\mathrm{R}}$, Eq. (3) should be replaced as

$$
\Psi(T) \rightarrow \Psi\left(T, \mu_{\mathrm{R}}\right) .
$$

This means that the $\theta$ integration in Eq. (11) should be evaluated with fixed $T$ and also $\mu_{\mathrm{R}}$. Therefore, we must 
consider the complex chemical potential in the calculation of the quark number holonomy, where the sign problem arises. At finite imaginary chemical potential $\left(\mu_{\mathrm{R}}=0\right)$, we can use the $\gamma_{5}$ Hermiticity,

$$
\operatorname{det} \mathcal{D}(\mu)=\operatorname{det}\left[\gamma_{5} \mathcal{D}(\mu) \gamma_{5}\right]=\left[\operatorname{det} \mathcal{D}\left(-\mu^{*}\right)\right]^{*},
$$

where $\mathcal{D}$ is the Dirac operator. Therefore, the sign problem does not matter at finite imaginary chemical potential, $\mu^{*}=-\mu$, when we calculate $\Psi(T)$. On the other hand, at finite real chemical potential, $\mu_{\mathrm{R}} \neq 0$ and $\theta=0$, the relation (12) can not help us, but the Lefschetz thimble path integral method [35-37] does. In Ref. [38], it is shown that this method leads the saddle-points which manifests the $\mathcal{C K}$ symmetry where $\mathcal{C}$ and $\mathcal{K}$ express the charge and the complex conjugation operator, respectively. The sign problem can be avoided by the $\mathcal{C K}$ symmetric saddle points and then the mean-field calculation of QCD effective models such as the PNJL model is extremely simplified [39,40]. Unfortunately, the $\mathcal{C K}$ symmetry is not preserved at finite complex chemical potential, $\mu_{\mathrm{R}} \neq 0$ and $\theta \neq 0$, when we calculate Eq. (11). Thus, the calculation becomes complicated even in the mean-field calculation of the QCD effective models. In this case, we should perform the matter-of-fact calculation based on the Lefschetz thimble path integral method. Actual challenge of the calculation will be shown elsewhere.

\section{SUMMARY}

In this paper, we have proposed a new quantity to describe the confinement-deconfinement transition based on topological properties of QCD in the imaginary chemical potential region. We call it the quark number holonomy, which is defined by the contour integral of the quark number susceptibility along the closed loop of $\theta$. The quark number holonomy seems to be similar to the Uhlmann phase which can be used to classify topological order at finite $T$ in the condensed matter physics.

The quark number holonomy can have a nonzero value above $T_{\mathrm{RW}}$ or $T_{\text {Beard, }}$, and it becomes zero below these temperatures. This behavior is related to the different realizations of the free-energy degeneracy above and below $T_{\mathrm{RW}}$. From the model independent analysis, we find that the quark number holonomy is proportional to $N_{\mathrm{c}}^{2}$ in the deconfined phase, while it does not in the confined phase if we determined the confinement-deconfinement temperature as the topological phase transition. Also, we have shown the behavior of the quark number holonomy in the $T \rightarrow \infty$ and the $m \rightarrow \infty$ limit as a landmark to help the future lattice QCD simulation.

We have discussed the similarity between the quark number holonomy and the dual quark condensate which is sometimes used to investigate the confinement-deconfinement transition. Calculations of the dual quark condensate has the uncertainty when the dynamical quark is taken into account, but the quark number holonomy does not have such uncertainty. This is the strong advantage of the quark number holonomy. Also, we have explained how the quark number holonomy can describe the confinementdeconfinement transition from the landscape of the effective potential. In the confined phase, the fluctuation is strong and then the $\mathbb{Z}_{N_{\mathrm{c}}}$ images collapse to one minimum. On the other hand, the $\mathbb{Z}_{N_{\mathrm{c}}}$ images withstand the fluctuation in the deconfined phase. Therefore, the quark number holonomy can describe the confinement-deconfinement transition via the free-energy degeneracy. Finally, we have discussed the sign problem when we calculate the quark number holonomy at finite real chemical potential. In this case, we should consider the complex chemical potential and, thus, we need to take good care of the sign problem.

\section{ACKNOWLEDGMENTS}

The authors thank M. Sato, whose suggestions for the quantum order-parameter of the topological phase transition at finite temperature motivated us to start this study. K. K. thanks H. Tsukiji for helpful comments. K. K. is supported by Grant-in-Aid No. 26-1717 from the Japan Society for the Promotion of Science (JSPS). A. O. is supported in part by the Grants-in-Aid for Scientific Research No. 15K05079, No. 15H03663 and No. 16K05350 from JSPS, Grants-in-Aid for Scientific Research on Innovative Areas (No. 2404: 24105001, 24105008) from MEXT: Ministry of Education, Culture, Sports, Science and Technology, and by the Yukawa International Program for Quark-Hadron Sciences.
[1] X. G. Wen, Int. J. Mod. Phys. B 04, 239 (1990).

[2] M. Sato, Phys. Rev. D 77, 045013 (2008).

[3] K. Kashiwa and A. Ohnishi, Phys. Lett. B 750, 282 (2015).

[4] A. Uhlmann, Rep. Math. Phys. 24, 229 (1986).

[5] O. Viyuela, A. Rivas, and M. A. Martin-Delgado, Phys. Rev. Lett. 112, 130401 (2014).
[6] A. Roberge and N. Weiss, Nucl. Phys. B275, 734 (1986).

[7] Y. Sakai, K. Kashiwa, H. Kouno, M. Matsuzaki, and M. Yahiro, Phys. Rev. D 79, 096001 (2009).

[8] K. Fukushima, Phys. Lett. B 591, 277 (2004).

[9] P. de Forcrand and O. Philipsen, Nucl. Phys. B642, 290 (2002). 


\section{KOUJI KASHIWA and AKIRA OHNISHI}

[10] M. D'Elia and M.-P. Lombardo, Phys. Rev. D 67, 014505 (2003).

[11] L.-K. Wu, X.-Q. Luo, and H.-S. Chen, Phys. Rev. D 76, 034505 (2007).

[12] Y. Nishida, Phys. Rev. D 69, 094501 (2004).

[13] N. Kawamoto, K. Miura, A. Ohnishi, and T. Ohnuma, Phys. Rev. D 75, 014502 (2007).

[14] D. J. Gross, R. D. Pisarski, and L. G. Yaffe, Rev. Mod. Phys. 53, 43 (1981).

[15] N. Weiss, Phys. Rev. D 24, 475 (1981).

[16] M. D'Elia and F. Sanfilippo, Phys. Rev. D 80, 111501 (2009).

[17] C. Bonati, G. Cossu, M. D'Elia, and F. Sanfilippo, Phys. Rev. D 83, 054505 (2011).

[18] K. Kashiwa and R. D. Pisarski, Phys. Rev. D 87, 096009 (2013).

[19] G. Aarts, E. Seiler, D. Sexty, and I.-O. Stamatescu, Phys. Rev. D 90, 114505 (2014).

[20] Y. Aoki, Z. Fodor, S. D. Katz, and K. K. Szabo, Phys. Lett. B 643, 46 (2006).

[21] M. Cheng et al., Phys. Rev. D 77, 014511 (2008).

[22] S. Borsanyi, Z. Fodor, C. Hoelbling, S. D. Katz, S. Krieg, C. Ratti, and K. K. Szabo (Wuppertal-Budapest), J. High Energy Phys. 09 (2010) 073.

[23] A. Bazavov et al., Phys. Rev. D 85, 054503 (2012).

[24] A. Miyahara, Y. Torigoe, H. Kouno, and M. Yahiro, arXiv:1604.05002.

[25] C. Bonati, M. D'Elia, M. Mariti, M. Mesiti, F. Negro, and F. Sanfilippo, Phys. Rev. D 93, 074504 (2016).
PHYSICAL REVIEW D 93, 116002 (2016)

[26] E. Bilgici, F. Bruckmann, C. Gattringer, and C. Hagen, Phys. Rev. D 77, 094007 (2008).

[27] E. Bilgici, F. Bruckmann, J. Danzer, C. Gattringer, C. Hagen, E. M. Ilgenfritz, and A. Maas, Few Body Syst. 47, 125 (2010).

[28] E. Bilgici, Ph.D. thesis, University of Graz, 2009, http:// physik.uni-graz.at/itp/files/bilgici/dissertation.pdf.

[29] C. S. Fischer, Phys. Rev. Lett. 103, 052003 (2009).

[30] K. Kashiwa, H. Kouno, and M. Yahiro, Phys. Rev. D 80, 117901 (2009).

[31] S. Benič, Phys. Rev. D 88, 077501 (2013).

[32] F. Marquez, A. Ahmad, M. Buballa, and A. Raya, Phys. Lett. B 747, 529 (2015).

[33] Z. Zhang and Q. Miao, Phys. Lett. B 753, 670 (2016).

[34] P. M. Lo, B. Friman, O. Kaczmarek, K. Redlich, and C. Sasaki, Phys. Rev. D 88, 074502 (2013).

[35] E. Witten, AMS/IP Stud. Adv. Math. 50, 347 (2011).

[36] M. Cristoforetti, F. Di Renzo, and L. Scorzato (Aurora Science Collaboration), Phys. Rev. D 86, 074506 (2012).

[37] H. Fujii, D. Honda, M. Kato, Y. Kikukawa, S. Komatsu, and T. Sano, J. High Energy Phys. 10 (2013) 147.

[38] Y. Tanizaki, H. Nishimura, and K. Kashiwa, Phys. Rev. D 91, 101701 (2015).

[39] H. Nishimura, M. C. Ogilvie, and K. Pangeni, Phys. Rev. D 90, 045039 (2014).

[40] H. Nishimura, M. C. Ogilvie, and K. Pangeni, Phys. Rev. D 91, 054004 (2015). 